\title{
The Statistical Analysis of Characteristics of Coronal Mass Ejection During the Descending Phase of Solar Cycle 23 and 24
}

\author{
Hemlata Dharmashaktu ${ }^{1 *}$ - N.K.Lohani ${ }^{2}$ \\ ${ }^{1}$ Dept. of Physics, Indira Priyadarshini Govt. Girls P.G. C.C Haldwani, Nainital \\ ${ }^{2}$ Department of Physics, Kumaun University Nainital \\ *Corresponding Author Email: hemlata.dharmashaktu@gmail.com
}

Received: 28.8.2021; Revised: 19.9.2021; Accepted: 24.9.2021

(C)Society for Himalayan Action Research and Development

\begin{abstract}
The characteristics of CMEs we studied are angular width, linear speed, and acceleration for all categories of $\mathrm{CMEs}$ such as narrow $\left(\mathrm{W} \leq 20^{\circ}\right)$, intermediate $\left(20^{\circ}<\mathrm{W}<200^{\circ}\right)$, wide $\left(\mathrm{W} \geq 200^{\circ}\right)$ and linear speed $<500 \mathrm{~km} / \mathrm{s}$ during the descending phase of solar cycle 23 and 24 and compared them. We have found that there are 1951 narrow CMEs during solar cycle 23 that is 1.9 times greater than in solar cycle 24 (1047). On the other side, the number of intermediate CMEs during solar cycle 24 (1571) is 1.14 times more than solar cycle 23 (1162). We observed no noticeable difference between the number of wide CMEs of solar cycle 23 (29) and 24 (36). The angular width of CMEs during the descending phase of solar cycle 23 and solar cycle 24 , predominately distributed around 100-600. The fascinating result is that the angular width distributions for the descending phase of solar cycles are approximately identical. On comparing the results of linear speed of both solar cycle, we can say that, (i) 93.7\% (1729) and 87.7\% (908) of narrow CMEs, (ii) 97\% (1328) and 94\% (1479) of intermediate CMEs and (iii) $44 \%$ (13) and 42\% (15) of wide CMEs have speed of $<500 \mathrm{~km} \mathrm{~s}-1$, respectively. Mostly the fractions of narrow and intermediate CMEs decline sharply at the speeds greater than $500 \mathrm{~km} \mathrm{~s}-1$. The maximum speed observed during the $23 \mathrm{rd}$ cycle is $1994 \mathrm{~km} / \mathrm{s}$ (wide CME) and the 24 th cycle is $3163 \mathrm{~km} / \mathrm{s}$ (wide CME) respectively. It was noticed that the speed of the 24th solar cycle CME is higher than the 23rd solar cycle CME. The major fraction of CMEs has acceleration in the range of -20 to $20 \mathrm{~km} \mathrm{~s}-2$, all types of CMEs. The narrow and intermediate CMEs mostly show acceleration while wide CMEs show deceleration.
\end{abstract}

Keywords: · Sun · Coronal mass ejections · Narrow · Intermediate · Wide · Linear speed.

\section{Introduction}

Coronal Mass Ejections (CMEs) are the most energetic phenomena in the solar atmosphere. CMEs were first detected in the coronagraph images in year 1971 using the Seventh Orbiting Solar Observatory (OSO-7) (Tousey 1973). Typical coronagraphs have an occulting disc to artificially eclipse the bright photosphere, so that the faint corona structures outside the periphery of the occulting disc can be viewed in the photospheric light scattered of these structures. CMEs are large scale magnetised plasma structures originating from closed magnetic regions on the sun, active regions, filament regions, active region complexes and trans equatorial interconnecting regions. CMEs have posed the severe threat for dynamic and variable conditions in upper atmosphere. Researches in past four decades identifies that CMEs are the most energetic phenomenon in the heliosphere (Gopalswamy 2006, Richardson et.al. 2000).

The CME is a large outburst of coronal magnetic field and typically $10^{9}$ to $10^{10}$ tons of plasma into interplanetary space at speeds of some $\mathrm{km} \mathrm{s}-1$ to $\sim 3000 \mathrm{~km} \mathrm{~s}-1$ with an average value of $483 \mathrm{~km} \mathrm{~s} \mathrm{-1} \mathrm{(Gopalswamy}$ et.al. 2004, Yashiro et.al. 2004). The frequency of CMEs varies with sunspot cycle. The occurrence of CMEs depends on the phase of the solar cycle. During solar maximum, CMEs originate from active regions and their 
occurrence rate may be 2-3 a day, while during solar minimum the occurrence rate may be one CME per week Gopalswami et al (Gopalswamy et.al. 2002, Gopalswamy et.al. 2003) suggested that only fast and wide CMEs have an important role in the production of large solar energetic particles (SEPs) whereas Kahlar et al (2001) stated that some impulsive SEP events were also associated with fast but narrow CMEs. Narrow CMEs may also play a vital role in compulsive SEP production or propagation into the interplanetary medium. So, it is significant to observe the characteristics and birth of narrow CMEs.

In 1998, 27 jet-like CMEs (having typical width of $\sim 2^{\circ}-4^{\circ}$ ) were investigated and observed above the polar coronal holes in the region of 3-4 solar radii $\left(R_{\mathrm{o}}\right)$, using the Extreme Ultraviolet Imaging Telescope (EIT) on board SOHO (Wang et al 1998). It is also observed that the leading edges of jet-like CMEs propagated at the speed of 400-1100 $\mathrm{km} \mathrm{s}-1$, while the bulk of their material travelled at around $250 \mathrm{~km} / \mathrm{s}$. Gilbert et al (2001) examined the properties of 15 narrow CMEs and found that the apparent speed of these CMEs are ranged from 159-630 km s-1 and they suggested that narrow CMEs had no visible internal structure (Wang et al 1998).

It is possible that some narrow CMEs do contain visible structure but the resolution of the LASCO instrument limits our ability to observe it. In other observation, stated that the narrow CMEs seem to be mass flows in vertical flux tubes. They investigated the properties of narrow CMEs and found that the occurrence of narrow CMEs increases towards solar maximum (Yashiro et al 2003). They showed that the average speed of narrow CMEs increases from $269 \mathrm{~km} \mathrm{~s}-1$ during solar minimum (1996-1997) to $545 \mathrm{~km} / \mathrm{s}$ at solar maximum (1999-2000) and they considered angular width $<20^{\circ}$ for narrow CMEs. Mittal et al (2009) investigated properties of narrow CMEs (width $\leq 20^{\circ}$ during the period of 19962007. They found some differences between narrow and normal CMEs. Yashiri et al (2008) compared the statistical properties of 23rd cycle CMEs by manual and automatic method, in which they excluded the CMEs with angular width $<30^{\circ}$ because such CMEs are highly subjective. In our present study, our primary motive is to investigate, the CMEs characteristics as speed, angular width, linear speed and acceleration of narrow, intermediate and wide CMEs during solar cycle 23 (20062008) and 24 (2016-2018) and to compare how the changes take place from one cycle to another among all three categories.

\section{Data and Methodology}

The data used in present study was obtained from the Solar and Heliospheric Observatory (SOHO) mission's Large Angle and Spectrometric Coronagraph (LASCO), (Brueckner et al 1995) as compiled in the CME catalogue

\section{http://cdaw.gsfc.nasa.gov/CME list;}

(Yashiroet al 2004, Gopalswamy et al 2009). The catalogue provides the CME data from 1996 to present day. The LASCO instrument consists of three coronagraphs $\mathrm{C} 1, \mathrm{C} 2$ and $\mathrm{C} 3$ 
that span the fields of view 1.1-3 $\mathrm{R}_{\mathrm{o}}, 2-6 \mathrm{R}_{\mathrm{o}}$ and 4-30 $R_{o}$, respectively (where, $R_{0}=$ solar radius $\left.=6.96 \times 10^{5} \mathrm{~km}\right)$. Both $\mathrm{C} 2$ and $\mathrm{C} 3$ are externally occulted white light coronagraphs; while $\mathrm{C} 1$ was designed for spectrometric purposes but has been out of operation since June 1998 (Wang and Sheeley 2009). All events in our study were divided into three categories: (i) narrow CMEs, with the angular width $\leq 20^{\circ}$ (Yashiro et al 2004, Mittal et al 2009); (ii) intermediate CMEs, with the angular width in the range $20^{\circ}<$ width $<200^{\circ}$ [17] and (iii) wide CMEs, with the angular width $\geq 200^{\circ}$ (Gopalswamy et al 2001). In our present study, we observed total 6005 CMEs events out of which 3351 events found from January 2006 to December 2008 and 2654 events from January 2016 to December 2018 during descending phase of the solar cycle 23 and 24 respectively.

\section{Results}

The main characteristics of CMEs studied by us are angular width, linear speed and acceleration for all categories of CMEs such as narrow, intermediate and wide. Each property is discussed one by one in following subsections-

\section{Angular Width}

The angular width of CMEs typically refers to the position angle extent in the sky plane in degree. For CMEs away from the limb, the measured width is likely to be an overestimate but it should be true width for CMEs originating from close to the limb. The angular width of CMEs projected in the plane of the sky ranges from $\sim 2^{\circ}$ to $360^{\circ} \mathrm{CMEs}$ originating from close to the limb resemble measured width as true width [11]. We have studied the distribution of angular width for the year 2006-2008 and 2016-2018. The observed numbered of CMEs of each year is shown on the plots fig. 1(a-f) and table 1. From these plots we observed that the pattern of distribution of angular width for years 2006, 2007, 2016 and 2017 were approximately same but years whereas the narrow CMEs for year 2008 and 2018 shows just opposite behaviour.

Table 1. Distribution of angular width during descending phase of solar cycle 23 and 24:

\begin{tabular}{ccccc} 
Year & Total & $\begin{array}{c}\text { Narrow } \\
\left(\boldsymbol{W} \leq \mathbf{2 0 ^ { \circ }}\right)\end{array}$ & $\begin{array}{c}\text { Intermediate } \\
\left(\mathbf{2 0}^{\circ}<\mathbf{W}^{2000^{\circ}}\right)\end{array}$ & $\begin{array}{c}\text { Wide } \\
\left(\mathbf{W}^{\circ} \mathbf{2 0 0 ^ { \circ }}\right)\end{array}$ \\
\hline 2006 & 1046 & $418(40 \%)$ & $606(58 \%)$ & $20(2 \%)$ \\
2007 & 1442 & $921(64 \%)$ & $515(36.6 \%)$ & $6(.4 \%)$ \\
2008 & 863 & $612(71 \%)$ & $248(28.7 \%)$ & $3(.3 \%)$ \\
CMEs (23SC) & 3351 & $1951(58 \%)$ & $1369(41 \%)$ & $29(1 \%)$ \\
2016 & 1392 & $551(40 \%)$ & $823(59 \%)$ & $18(1 \%)$ \\
2017 & 786 & $282(36 \%)$ & $489(62 \%)$ & $15(2 \%)$ \\
2018 & 476 & $214(45 \%)$ & $259(54 \%)$ & $3(1 \%)$ \\
CMEs (24SC) & 2654 & $1047(39.4 \%)$ & $1571(59.2 \%)$ & $36(1.4 \%)$ \\
\hline
\end{tabular}




\section{Vol. 16(2) Special Issue ICRAMS-2021, (2021), 61-69}
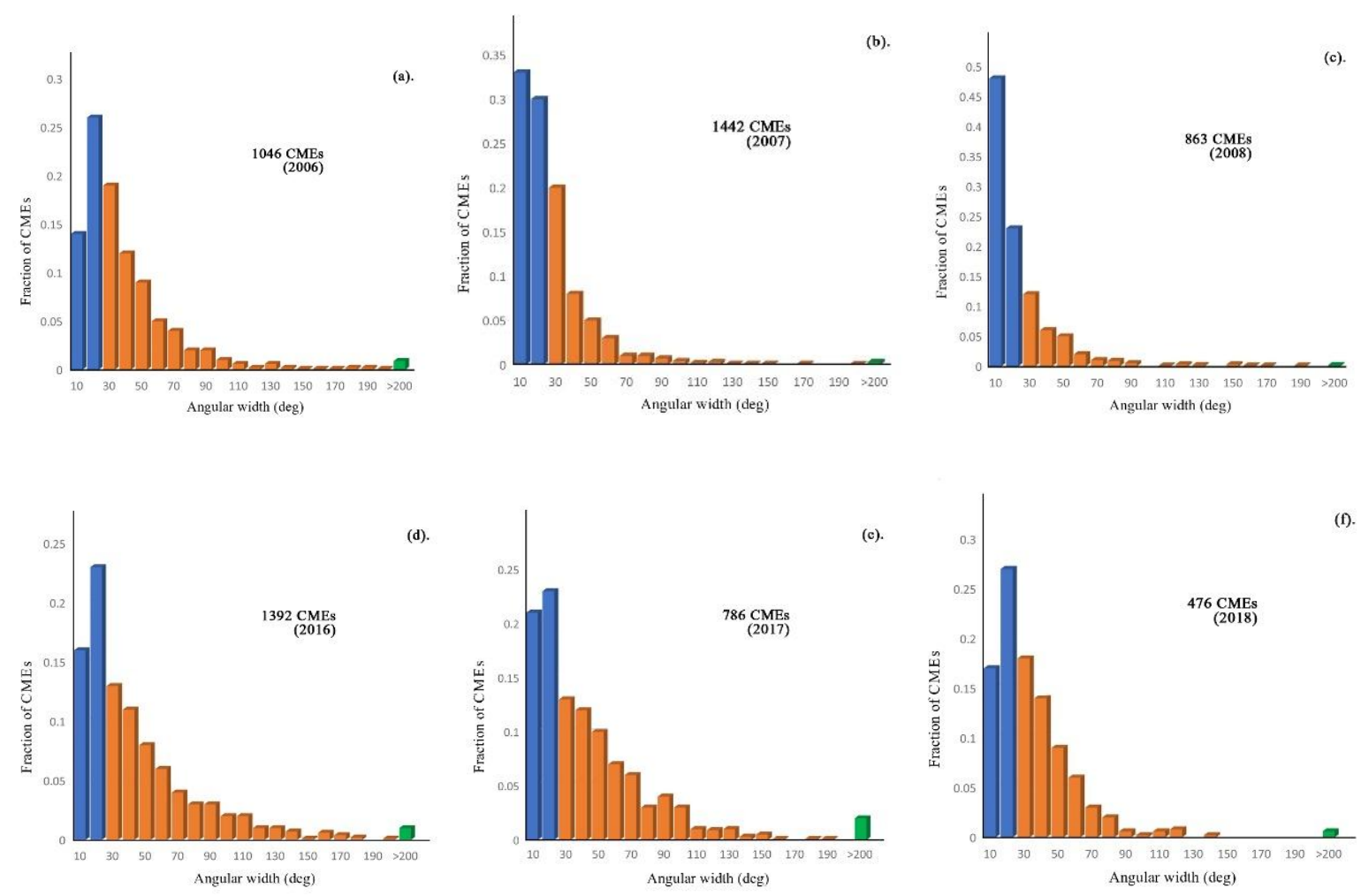

Fig.1 Annual distribution of angular width of narrow (blue), intermediate (orange) and wide (green) CMEs for period 2006-2008 and 2016-2018 of descending phase of solar cycle 23 and 24. Each bin equals to $10^{\circ}$ in $\mathrm{X}$-axis and CMEs fraction 0.05 in $\mathrm{Y}$-axis.

We studied 3351 and 2654 CME events during descending phase of solar cycle 23 and 24 respectively. The plots are shown in figure 2 . During descending phase of solar cycle 23, 58\% CMEs whereas in solar 24, 39.4\% CMEs are narrow. However, there is $41 \%$ CMEs during solar cycle 23 whereas $59 \%$ CMEs are intermediate during solar cycle 24 . There is no noticeable difference between the number of wide CMEs of solar cycle $23(1 \%)$ and 24 (1.4\%). The angular width of CMEs predominately distributed around $10^{\circ}-30^{\circ}$ whereas during solar cycle 24 , around $10^{\circ}-50^{\circ}$. The fig. 1 and 2 also shows that most of the
CMEs observed in both solar cycles have lower angular width distribution. Similar results were found in 2007[12][17]. Also,[13] found there is a significant contrast between narrow CMEs rate using CDAW and CACTUS records. Since the CMEs are spotted manually, there is always the probability that some CMEs are skipped. Furthermore, it is probably that some narrow CMEs are missed due to the consequence of visibility issues. They also estimated that $20 \%$ of CMEs may not have been detected by LASCO because they are either disguised by the occulting ring or they occur at back sided of visibility. 


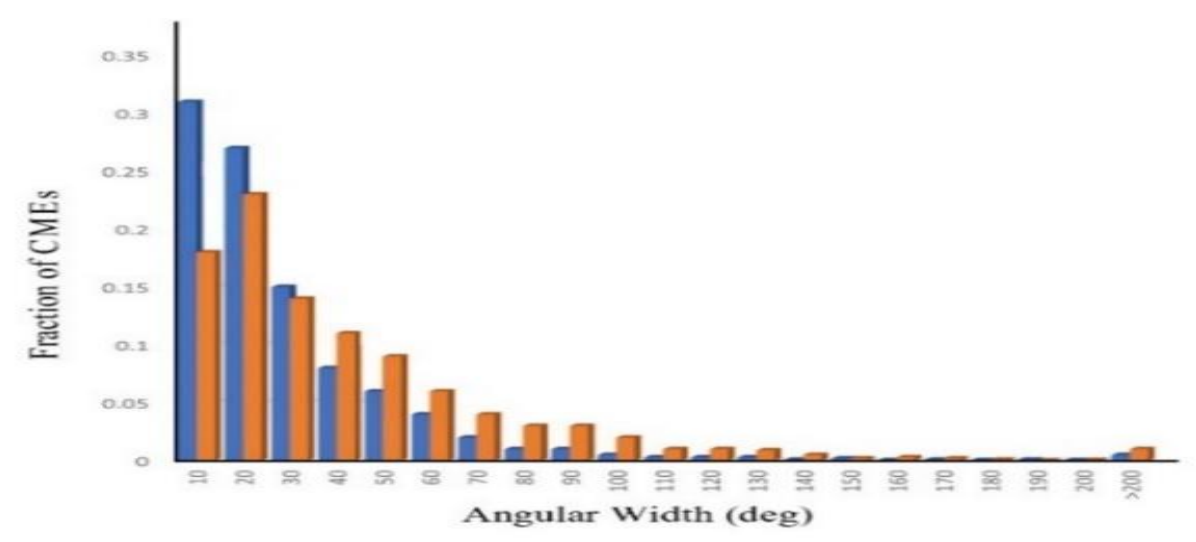

Fig.2 Distribution of angular width of CMEs during descending phase of solar cycle 23 (blue bar) and 24 (orange bar). Each bin equals to $10^{\circ}$ in $\mathrm{X}$-axis and CMEs fraction 0.05 in $\mathrm{Y}$ - axis.

\section{Linear speed}

Mass motion is a basic characteristic of CMEs and quantified by their speeds. Coronagraphs obtain images with a preset time cadence and when a CME occurs, the leading edge moves to a greater heliocentric distance. On measuring the heliocentric distance of the leading edge of a CME in each LASCO image, one obtains CME height as a function of time. Height-time measurements are made in the sky plane, so all the derived parameters such as speed etc are the lower limits to the real values [2]. The height-time plots are then fitted to first order polynomials which give an average speed within the LASCO field of view. The CME velocity general means the radial propagation speed of the top part of a CME frontal loop.

Fig.3 shows the annual linear speed distribution of narrow (blue bars), intermediate (orange bar) and wide (green bars) CMEs from 2006-2007 and 2016-2018. We have excreted 3343 CMEs events out of 3351 and 2654
CMEs during descending phase of solar cycle 23 and 24, respectively. SOHO/LASCO is unable to measure some CME speeds due to the poor occurrence, therefore the 8 CMEs events were excluded that used in the width distribution. It is observed that, the $93.7 \%$ (1729) of narrow CMEs in solar cycle 23 and $87.7 \%$ (1328) of narrow CMEs in solar cycle 24 having the speed $>500 \mathrm{~km} / \mathrm{s}$. The number of CMEs during a descending phase of solar cycle 23 is 1.9 times greater than that of solar cycle 24 . The average speed of solar cycle 24 (315 km s-1) is slightly greater than its previous solar cycle (293 km s-1). The number of intermediate CMEs having speed $>500 \mathrm{~km}$ s-1 is $97 \%$ (1328) during solar cycle 23 and 94\% (1479) during solar cycle 24. The maximum speed of intermediate CMEs during solar cycle $23(1398 \mathrm{~km} \mathrm{~s}-1)$ is greater than that of solar cycle $24(1118 \mathrm{~km} / \mathrm{s})$. The maximum speed of narrow CMEs during solar cycle $23(928 \mathrm{~km} / \mathrm{s})$ is less than the solar cycle $24(1101 \mathrm{~km} \mathrm{~s}-1)$. The speed distribution of 
wide CMEs having speed > $500 \mathrm{~km} \mathrm{~s}-1$ is $44 \%$ (13) during solar cycle 23 and $42 \%$ (15) during solar cycle 24 . The maximum speed of wide CMEs during solar cycle $24(3163 \mathrm{~km} \mathrm{~s}-$ 1) is much greater than solar cycle 23 (1994 $\mathrm{km} \mathrm{s}-1)$. The average speed of narrow and wide CMEs during solar cycle 24 (315 and $657 \mathrm{~km} \mathrm{~s}-1)$ is greater than solar cycle 23 (293 and $583 \mathrm{~km} \mathrm{~s}-1)$. Whereas there is no noticeable difference between the speed of intermediate CMEs for solar cycle $23(252 \mathrm{~km}$ $\mathrm{s}-1)$ and solar cycle 24 (255 km s-1). The average speed of all CMEs for solar cycle 23 and 24 is 275 and $288 \mathrm{~km} \mathrm{~s}-1$. Gopalswami et al [7] stated that the median and average speeds during solar minimum (1996-1997) were 237 and $269 \mathrm{~km} \mathrm{~s}-1$ for narrow CMEs whereas 257 and $299 \mathrm{~km} \mathrm{~s}-1$ for normal CMEs. [12] also stated that the median and average speeds during solar minimum (19961998, 2005-2007) were 309 and 337 km s-1 for narrow CMEs whereas 282 and $334 \mathrm{~km} \mathrm{~s}-1$ for normal CMEs.

Most of the narrow and intermediate CMEs have speeds in the range of $250-500 \mathrm{~km} \mathrm{~s}-1$. The corresponding speeds for wide CMEs lie in the range of $250-1000 \mathrm{~km} \mathrm{~s}-1$. From fig 3 it is also observed that the occurrence rate of narrow and intermediate CMEs declines sharply at linear speeds $<500 \mathrm{~km} / \mathrm{s}$, whereas the distribution of wide CMEs has a high speed with declining occurrence rate. On comparing the speeds of two phases the CMEs speed of 24th solar cycle is higher than that of 23rd solar cycle. In contrast to results of Laxmi et al [17] stated that the speed of CMEs in solar cycle 24 is smaller than solar cycle 23.
The maximum speed observed during 23rd cycle is $1994 \mathrm{~km} \mathrm{~s}-1$ (wide CME) and the maximum speed observed during 24th cycle is 3163 km s-1 (wide CME).

\section{Acceleration:}

Figures 4 (a-f) shows the annual distribution of acceleration from 2006-2008 and 2016-2018 for narrow, intermediate and wide CMEs for descending phase of solar cycle 23 and 24 respectively. The quadratic fit to the heighttime plot gives constant acceleration which is an approximation because the acceleration may change with time [2][12]. All CMEs have positive acceleration as they first lift off from rest. It is clear from figures 4 that during both solar cycle narrow and intermediate CMEs are biased towards acceleration, but a small portion of both the narrow and intermediate CMEs do move with deceleration. The wide CMEs mostly show deceleration whereas small portion do move with positive acceleration.

\section{Conclusion}

We have analysed 3351 and 2654 CMEs having width $\leq 20^{\circ}$ (narrow), $20^{\circ}<$ Width $<$ $200^{\circ}$ (intermediate) and $\geq 200^{\circ}$ (wide) CMEs observed for the period 2006-2008 and 20162018 during the descending phase of solar cycle 23 and 24 and summarized the following results:

1. In our study, we found that there are 1951 narrow CMEs during solar cycle 23 and that is 1.9 times greater than in solar cycle 24 (1047). On the other side, the number of intermediate CMEs during solar cycle 24 (1571) is 1.14 
times more than solar cycle 23 (1162). We observed no noticeable difference between the number of wide CMEs of solar cycle 23 (29) and 24 (36). The angular width of CMEs during descending phase of solar cycle 23 and solar cycle 24, predominately distributed around $10^{\circ}-60^{\circ}$. The fig. 1 and 2 also shows that most of the CMEs observed in both solar cycles have lower angular width distribution.
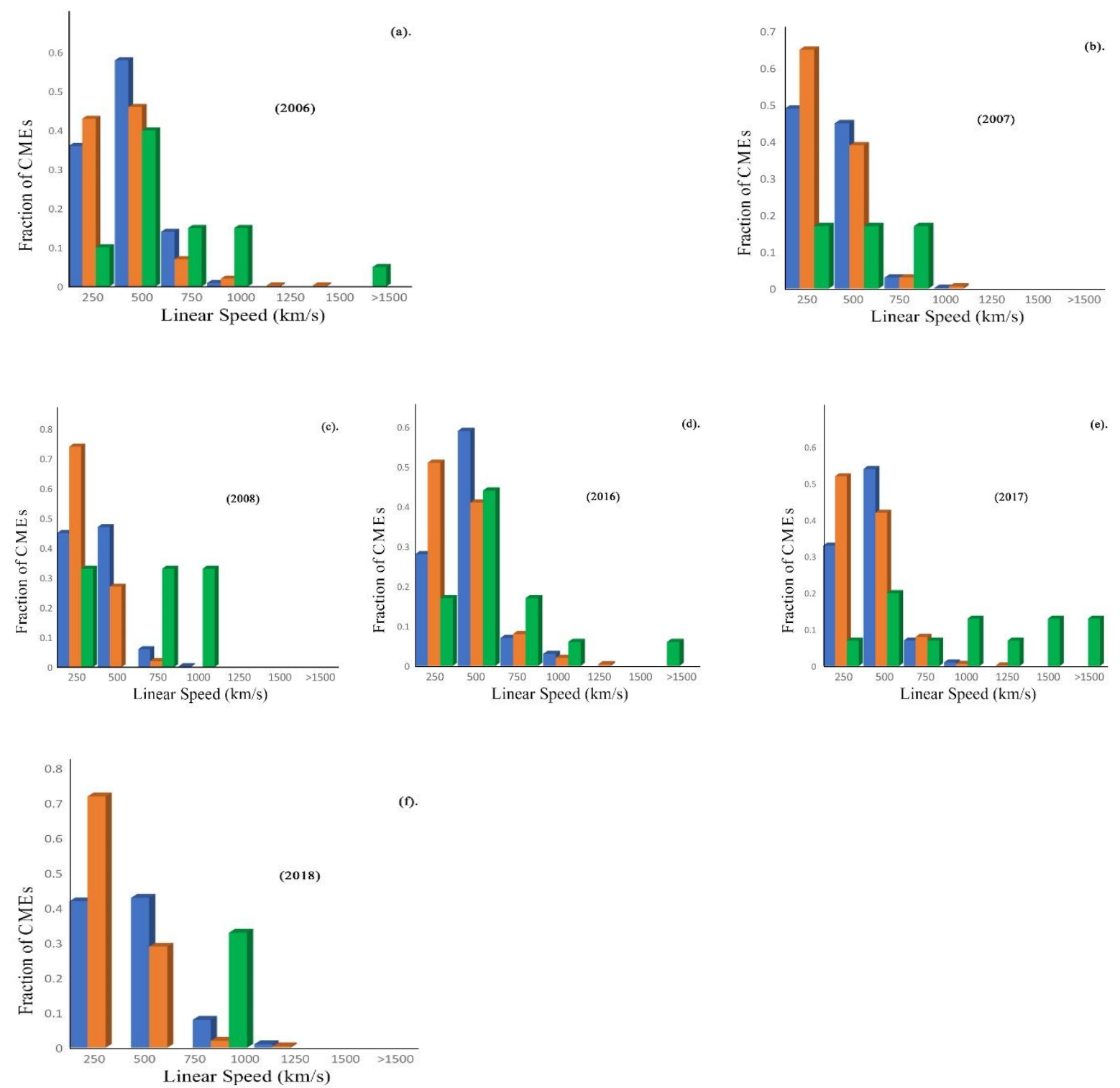

Fig.3 Annual distribution of linear speed of narrow (blue bar), intermediate (orange bar) and wide (green bar) CMEs for period 2006-2008 and 2016-2018 of descending phase of solar cycle 23 and 24. Each bin equals to $250 \mathrm{~km} / \mathrm{s}$ in $\mathrm{X}$-axis and CMEs fraction 0.1 in $\mathrm{Y}$-axis.

2. The average speeds of narrow and wide CMEs during descending phase of solar cycle 24 (315 and $657 \mathrm{~km} \mathrm{~s}-1)$ are more than those values (293and $583 \mathrm{~km} \mathrm{~s}-1$ ) during solar cycle
23. There is no noticeable difference in average speeds of intermediate CMEs 252 and $255 \mathrm{~km} \mathrm{~s}-1$ during solar cycle 23 and 24 respectively. On comparing the results of both 
solar cycle, we can say that : (i) $93.7 \%$ (1729) and $87.7 \%$ (908) of narrow CMEs, (ii) $97 \%$ (1328) and 94\% (1479) of intermediate CMEs and (iii) $44 \%$ (13)and $42 \%$ (15) of wide CMEs have speed $<500 \mathrm{~km} \mathrm{~s}-1$, respectively. Most of the narrow and intermediate CMEs have speeds in the range of $250-500 \mathrm{~km} \mathrm{~s}-1$. The corresponding speeds for wide CMEs lie in the range of 250-1000 km s-1. Mostly the fraction of narrow and intermediate CMEs declines sharply at speeds greater than $500 \mathrm{~km} \mathrm{~s}-1$.
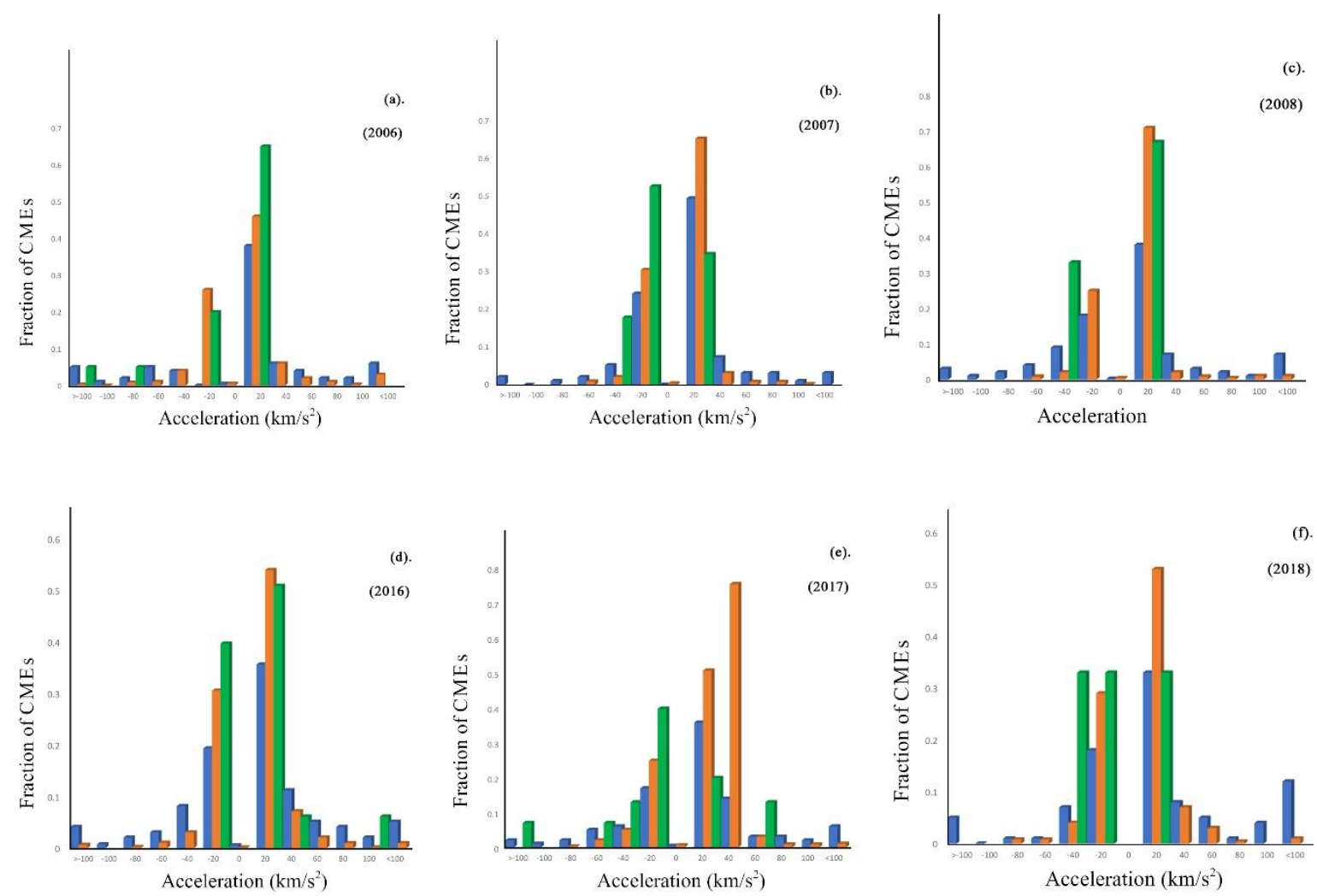

Fig.4 Annual distribution of acceleration of narrow (blue bar), intermediate (orange bar) and wide (green bar) CMEs for period 2006-2008 and 2016-2018 of descending phase of solar cycle 23 and 24. Each bin equals to $20 \mathrm{~km} \mathrm{~s}-2$ in $\mathrm{X}$-axis and CMEs fraction 0.1 in $\mathrm{Y}$-axis.

3. The maximum speed of CMEs during solar cycle $24(3163 \mathrm{~km} \mathrm{~s}-1)$ is more than during solar cycle 23 (1994 km s-1).

4. The major fraction of CMEs has acceleration in the range of -20 to $20 \mathrm{~km} \mathrm{~s}-2$, for narrow, intermediate and wide CMEs. The narrow and intermediate CMEs mostly shows acceleration while wide CMEs shows deceleration.
From our above study we reach at the conclusion that there are some differences in results of narrow, intermediate and wide CMEs with previous results [17].

\section{Acknowledgement}

Authors would like to thank an anonymous referee for providing fruitful comments and suggestions on the paper. The authors greatly acknowledge the data support provided by SOHO/LASCO CME catalogue. We are 
thankful to our college authorities for their help and encouragement.

\section{References}

Tousey, R., The solar corona; Space Res., 13 (2), 713-730, (1973).

Gopalswamy, N., Coronal Mass Ejections of Solar Cycle 23; J. Astrophys. Astr. 27,243-254 (2006).

Richardson, I. G., Berdichevsky, D., Desch, M. D., Farrugia, C. J., Solar-cycle variation of low- density solar wind during more than three solar cycles; Geophys. Res. Lett., 27, 23,3761 (2000).

Gopalswamy, N., Poletto, G., Suess, S.T. (eds.), In the Sun and the Heliosphere as an Integrated System; p. 201. Kluwer Academic, Norwell (2004).

Yashiro, S., Gopalswamy, N., Michalek, G., St. Cyr, O.C., Plunkett, S.P., Rich, N.B., Howard, R.A., A Catalog of White Light Coronal Mass Ejections Observed by the SOHO Spacecraft; J. Geophys. Res., 109, A07105, (2004).

Gopalswamy, N., Yashiro, S., Michalek, G., Kaiser, M.L., Howard, R.A., Reames, D.V., Leske, R., von Rosenvinge, T.,Interacting coronal mass ejections and solar energetic particles; Astrophys. J. 572, L103 (2002).

Gopalswamy, N., Shimojo, M., Lu, W., Yashiro, S., Shibasaki, K., Howard, R.A., Prominence eruptions and coronal mass ejection: A statistical study using microwave observations; Astrophys. J. 586, 562 (2003).

Kahler, S.W., Reames, D.V., Sheeley, N.R. Coronal Mass Ejections Associated with I mpulsive

Solar Energetic Particle Events; Astrophys. J. 562, 558 (2001).

Wang, Y.M., Sheeley, N.R. Jr., Socker, D.G., Howard, R.A., Brueckner,G.E., Michels, D.J.,Moses, D., $\quad$ St. $\quad$ Cyr, O.C.,
Llebaria, A., Delaboudiniere, J.P.,Observations of correlated white-light and extreme-ultraviolet jets from polar coronal holes; Astrophys. J. 508, 899 (1998).

Gilbert, H.R., Serex, E.C., Holzer, T.E., MacQueen, R.M., McIntosh, P.S., Narrow Coronal Mass Ejections; Astrophys. J. 550, 1093 (2001).

Yashiro, S., Gopalswamy, N., Michalek, G., Howard, R.A., Properties of Narrow Coronal Mass Ejections Observed With LASCO; Adv. Space Res. 32, 2631 (2003).

Mittal, N., Pandey, K., Narain, U., Sharma, S.S., On properties of narrow CMEs observed with SOHO/LASCO; Astrophys. Space Sci. 323, 135 (2009).

Yashiro, S., Michalek, G., Gopalswamy, N., A comparison of coronal mass ejections identified by manual and automatic methods; Ann. Geophys. 26, 3103 (2008).

Brueckner, G.E., Howard, R.A., Koomen, M.J., Korendyke, C.M., Michels, D.J., Moses, J.D., Socker, D.G., Dere, K.P., Lamy, P.L., Llebaria, A., Bout, M.V., Schwenn, R., Simnett, G.M., Bedford, D.K., Eyles, C.J. The Large Angle Spectroscopic Coronagraph (LASCO); Solar Physics, 162, 357402(1995).

Gopalswamy, N., Yashiro, S., Michalek, G., Stenborg, G., Vourlidas, A., Freeland, S., Howard, R.A.: $\quad$ Earth Moon Planets 104(1), 295 (2009). 16. Wang, Y.M., Sheeley, N.R. Jr.: Astrophys. J. 575, 542 (2002).

Laxmi, M., A., Umapathy, S., Prakash, O., Vasanth, V., Studies on some properties of coronal mass ejections based on angular width; Astrophys Space Sci 335:373-378 (2011).

Gopalswamy, N., Yashiro, S., Kaiser, M. L., Howard, R. A., Bougeret, J.,Characteristics of coronal mass ejections associated with long-wavelength type II radio bursts; J. Geophys. Res., 106, A12, 29219 (2001). 